\title{
Calculation of the filtration problem by finite differences methods
}

\author{
Yuri Osipov*, Galina Safina and Yuri Galaguz \\ Moscow State University of Civil Engineering, Yaroslavskoe shosse, 26, Moscow, 129337, Russia
}

\begin{abstract}
The filtration problem of a suspension in a porous medium is relevant for the construction industry. In the design of hydraulic structures, construction of waterproof walls in the ground, grouting the loose soil, it is necessary to calculate the transfer and deposition of solid particles by the fluid flow. A one-dimensional filtration problem of a monodisperse suspension in a porous medium with a size-exclusion capture mechanism is considered. It is assumed that as the deposit grows, the porosity and admissible flow of particles through the porous medium change. The solution of the initial filtration model and the equivalent equations are calculated. For the numerical calculation of the problem, both standard first-order finite difference formulas and more accurate second-order schemes were used. The obtained solutions are compared with the results given by the TVD-scheme.
\end{abstract}

\section{Introduction}

The problems associated with the transport of small solid particles by the fluid flow and the deposition of particles in the pores of a porous medium are relevant for many technologies and industries. Wastewater and drinking water treatment, usage of industrial filters, construction of waterproof partitions for hazardous waste storage facilities, and grouting the loose soil by pumping a watery grout require solution of the filtration problems of a suspension in a porous medium $[1,2]$.

The deep bed filtration of the monodisperse suspension in a homogeneous porous medium is considered in the paper. Depending on the properties of the suspension and the porous medium, mechanical interaction, diffusion, viscosity, electrostatic and gravitational forces can play an important role in particles capture [3-5]. If the sizes of particles and pores are of the same order, then in many cases the mechanical-geometric mechanism of particle capture called the size-exclusion prevails: solid particles pass freely through largediameter pores and get stuck in pores whose diameter is smaller than the particle size [6].

A traditional mathematical model determining the one-dimensional filtration of an incompressible monodisperse suspension in a porous medium with a size-exclusion mechanism of particles capture relates the concentrations of suspended and retained particles to a system of two first-order partial differential equations. The equation of mass balance of suspended and retained particles is an analog of the continuity equation. The

\footnotetext{
* Corresponding author: yuri-osipov@mail.ru
} 
kinetic equation determines the increase of deposit concentration [7]. More complex models consider the change in the properties of the porous medium when deposit accumulates [8].

A lot of papers are devoted to the solution of the filtration problems. In a number of important cases it is possible to obtain exact and asymptotic solutions [7, 9, 10]. The methods of experimental and numerical modeling are developing actively [11-13].

The standard finite difference method for numerical solution of filtration problems is the replacement of partial derivatives by difference relations. Replacing differential equations by difference equations allows to construct an explicit difference scheme with first-order approximation. The disadvantages of this scheme are a small step in time for the fulfillment of the convergence condition and the low accuracy of the numerical solution, especially near the concentrations front where the solution is discontinuous. The use of more complex counter-current schemes and rapidly converging Lax-Vendroff schemes leads to unjustified smoothing or non-physical oscillations of the solution near the line of discontinuity [14]. These difference schemes, as well as TVD-schemes (Total Variation Diminishing scheme) in application to filtration problems have the first order of approximation [15].

To increase the accuracy of numerical calculations, an explicit difference scheme of the second order is constructed in this paper. It is used to solve both the initial system of differential equations of the filtration problem, and the equivalent first-order equation obtained in [10]. These numerical solutions are compared with the solutions based on the standard first-order schemes and the TVD scheme.

\section{Mathematical model}

A dimensionless model of filtration that considers the change of porous medium properties with the deposit accumulation is set in the domain

$$
\Omega=\{0<x<1, t>0\}
$$

by a system of mass transfer and deposit growth equations

$$
\begin{gathered}
\frac{\partial(g(S) C+S)}{\partial t}+\frac{\partial(f(S) C)}{\partial x}=0, \\
\frac{\partial S}{\partial t}=\Lambda(S) C .
\end{gathered}
$$

Here unknowns $C(x, t), S(x, t)$ are volumetric concentrations of suspended and retained particles, $\Lambda(S)$ is the filtration coefficient, functions $g(S), f(S), \Lambda(S)$ are smooth and positive for $S \geq 0$.

For the uniqueness of the solution of the system (1), (2), the boundary and initial conditions are set

$$
\begin{aligned}
& \left.C(x, t)\right|_{x=0}=1 ; \\
& \left.C(x, t)\right|_{t=0}=0 ; \\
& \left.S(x, t)\right|_{t=0}=0 .
\end{aligned}
$$


The condition (3) corresponds to the injection of a suspension with a constant unit concentration of suspended particles; the conditions (4) and (5) mean that at the initial moment the porous medium does not contain any suspended and retained particles.

It follows from the inconsistency of conditions (3) and (4) at the origin that the solution $C(x, t)$ has a discontinuity on the concentration front

$$
\Gamma: t=\frac{g(0)}{f(0)} x
$$

the solution $S(x, t)$ is continuous in the domain $\Omega$. The concentration front moves in a porous medium with constant velocity $v=f(0) / g(0)$ and divides $\Omega$ into two subdomains

$$
\Omega_{S}=\{0<x<1, t>x\} ; \Omega_{0}=\{0<x<1,0<t<x\} .
$$

In the domain $\Omega_{0}$ the solution is zero: $C=0 ; S=0$; in $\Omega_{S}$ the solution is positive: $C>0 ; S>0$.

The analytic solution of the problem (1)-(5) at the inlet of porous medium $x=0$ was obtained in [10] in an implicit form

$$
\int_{0}^{S(0, t)} \frac{d S}{\Lambda(S)}=t
$$

The classical filtration model with unchanging properties of a porous medium $g(S)=1, f(S)=1$ and a linear filtration coefficient $\Lambda(S)=a-b S$

$$
\frac{\partial C}{\partial t}+\frac{\partial C}{\partial x}+\frac{\partial S}{\partial t}=0 ; \quad \frac{\partial S}{\partial t}=(a-b S) C
$$

with conditions (3)-(5) has an exact solution in the domain $\Omega_{S}$

$$
C(x, t)=\frac{e^{b(t-x)}}{e^{b(t-x)}+e^{a x}-1} ; \quad S(x, t)=\frac{a}{b} \cdot \frac{e^{b(t-x)}-1}{e^{b(t-x)}+e^{a x}-1} .
$$

Solution (7), (9) is used for approbation of the numerical solution in $\Omega_{S}$.

The problem (1)-(5) can be reduced to a single 1-order equation for the function $S(x, t)$

$$
g(S) \frac{\partial S}{\partial t}+f(S) \frac{\partial S}{\partial x}+S \Lambda(S)=0
$$

with the condition (7). For the known $S(x, t)$ the solution $C(x, t)$ is determined from equation (2).

\section{Finite difference schemes of the filtration problem}

Finite difference schemes of the filtration problem (1)-(5) are constructed in a bounded domain $\Omega_{X T}=\{(x, t) \mid 0 \leq x \leq 1,0 \leq t \leq T\}$ on a rectangular grid with step $h$ in coordinate $x$ and $\tau$ in time $t$ : 


$$
x_{i}=i h, i=0,1,2, \ldots, N, h=1 / N ; t_{j}=j \tau, j=0,1,2, \ldots, M, \quad \tau=T / M
$$

The replacement of partial derivatives by the simplest finite differences

$$
\frac{\partial C}{\partial t} \rightarrow \frac{C_{i, j}-C_{i, j-1}}{\tau} ; \frac{\partial C}{\partial x} \rightarrow \frac{C_{i, j}-C_{i-1, j}}{h} ; \frac{\partial S}{\partial t} \rightarrow \frac{S_{i, j}-S_{i, j-1}}{\tau} ; \frac{\partial S}{\partial x} \rightarrow \frac{S_{i, j}-S_{i-1, j}}{h}
$$

specifies a standard finite difference scheme of the first-order approximation.

The relationship between step $\tau$ in time and step $h$ in coordinate $x$ is chosen using the Courant convergence condition

$$
\tau \leq \alpha h, \quad \alpha=\min _{S \geq 0}(g(S) / f(S)) .
$$

To construct the original finite difference schemes of the second order, the integration of equations (1), (2) and (9) over a rectangular cell of grid was used. It was found that when the integrals are approximated by the second-order formulas for the standard model (8), the trapezoid formula gives a more accurate result than the method of rectangles. Therefore, for construction of finite difference schemes for equations (1), (2), the trapezoid formula was used. For example,

$$
\begin{aligned}
& \int_{x_{i-1}}^{x_{i}} \int_{\tau_{j-1}}^{\tau_{j}} \frac{\partial(g(S) C)}{\partial \tau} d \tau d x=\int_{x_{i-1}}^{x_{i}} g\left(S\left(x, \tau_{j}\right)\right) C\left(x, \tau_{j}\right)-g\left(S\left(x, \tau_{j-1}\right)\right) C\left(x, \tau_{j-1}\right) d x= \\
& =\frac{h}{2}\left(g\left(S\left(x_{i}, \tau_{j}\right)\right) C\left(x_{i}, \tau_{j}\right)-g\left(S\left(x_{i}, \tau_{j-1}\right)\right) C\left(x_{i}, \tau_{j-1}\right)+\right. \\
& \left.+g\left(S\left(x_{i-1}, \tau_{j}\right)\right) C\left(x_{i-1}, \tau_{j}\right)-g\left(S\left(x_{i-1}, \tau_{j-1}\right)\right) C\left(x_{i-1}, \tau_{j-1}\right)\right)+O\left(h^{3}\right) ; \\
& \quad \int_{x_{i-1}}^{x_{i}} \int_{j-1}^{\tau_{j}} \Lambda(S) C d \tau d x=\frac{h \tau}{4}\left(\Lambda\left(S\left(x_{i}, \tau_{j}\right)\right) C\left(x_{i}, \tau_{j}\right)+\Lambda\left(S\left(x_{i}, \tau_{j-1}\right)\right) C\left(x_{i}, \tau_{j-1}\right)+\right. \\
& \left.+\Lambda\left(S\left(x_{i-1}, \tau_{j}\right)\right) C\left(x_{i-1}, \tau_{j}\right)+\Lambda\left(S\left(x_{i-1}, \tau_{j-1}\right)\right) C\left(x_{i-1}, \tau_{j-1}\right)\right)+O\left(h^{3}\right) .
\end{aligned}
$$

The predictors at the node $\left(x_{i}, t_{j}\right)$ are

$$
\tilde{C}_{i, j}=C_{i, j-1}+C_{i-1, j}-C_{i-1, j-1} ; \tilde{S}_{i, j}=S_{i, j-1}+S_{i-1, j}-S_{i-1, j-1},
$$

approximating the solution with accuracy $O\left(h^{2}\right)$.

The trapezium method is also used for numerical solution of equation (10). The solution $C(x, t)$ is obtained from equation (2) by the finite difference scheme of the second order

$$
C(x, t)=\frac{\partial S / \partial t}{\Lambda(S)}
$$

\section{Numerical calculation}

Numerical calculation of the filtration problem (1)-(5) is performed with steps $h=0.01$ and $\tau=g(0) h / f(0)$ for the equation coefficients measured in laboratory experiments for particles of radius $r=3.168 \mu \mathrm{m}[10]$ 


$$
\begin{aligned}
& g_{\exp }(S)=0.7635+2.44 \cdot 10^{-15} S+3.2 \cdot 10^{-14} S^{2}+3.6 \cdot 10^{-13} S^{3}, \\
& f_{\exp }(S)=0.9075+2.315 \cdot 10^{-4} S+2.27 \cdot 10^{-8} S^{2}-3.42 \cdot 10^{-8} S^{3}, \\
& \Lambda_{\exp }(S)=1.551-3.467 \cdot 10^{-3} S-1.16 \cdot 10^{-6} S^{2}-1.16 \cdot 10^{-7} S^{3} .
\end{aligned}
$$

In Figures 1 and 2 the graphs of suspended and retained particles concentrations at the porous medium outlet are shown. The lines correspond to the solutions of five different finite difference schemes of the filtration problem: first-order scheme for the system (1), (2) (solid line) and the equations (10), (12) (dash-dotted line); second-order scheme for the system (1), (2) (dash line) and the equations (10), (12) (dotted line); TVD-scheme (dashdotted with double dots line).

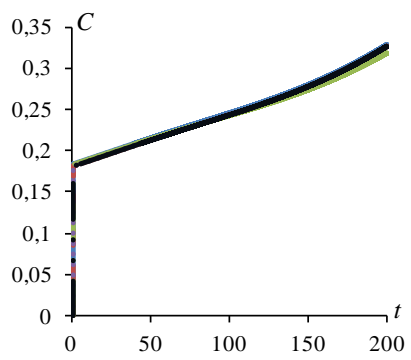

a)

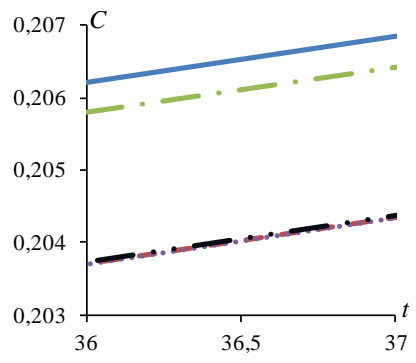

b)

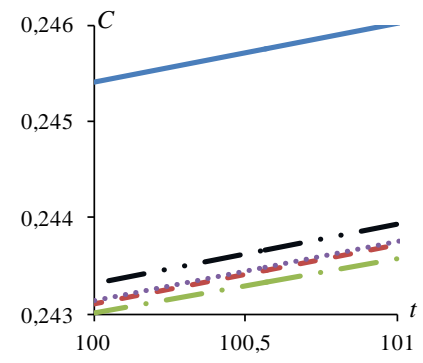

c)

Fig. 1. The suspended particles concentration $\left.C(x, t)\right|_{x=1}$ at the porous medium outlet

a) low resolution $0 \leq t \leq 200$;

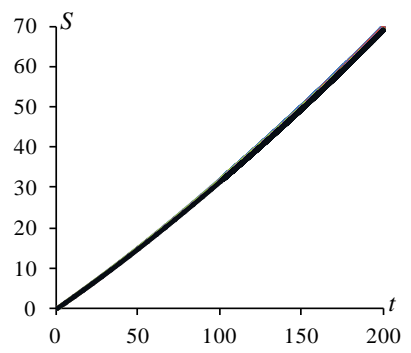

a) b) high resolution $36 \leq t \leq 37$;

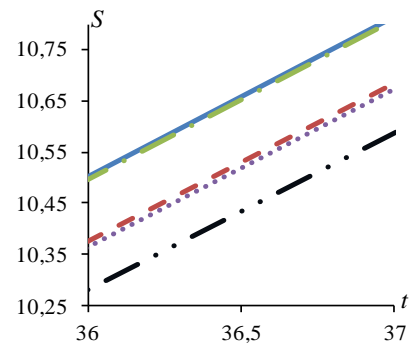

b) c) high resolution $100 \leq t \leq 101$.

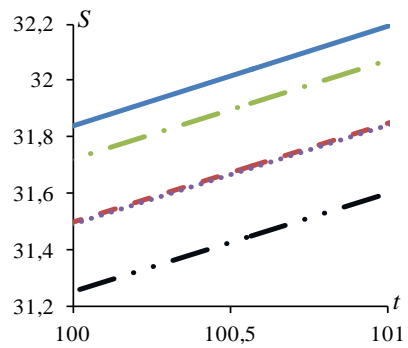

c)

Fig. 2. The retained particles concentration $\left.S(x, t)\right|_{x=1}$ at the porous medium outlet

a) low resolution $0 \leq t \leq 200$;

b) high resolution $36 \leq t \leq 37$;

c) high resolution $100 \leq t \leq 101$.

In Fig. 1 a), 2 a) with low resolution all five graphs merge into one line. Fig. 1 b), c) and $2 \mathrm{~b}), \mathrm{c})$ with high resolution show the mutual arrangement of the graphs.

\section{Conclusion}

The complex filtration model (1)-(5) does not have a simple analytical solution, so the problem is calculated numerically. In this paper, the comparison of the solutions at the porous medium outlet obtained by the finite difference methods of various accuracy is performed. It is shown that all solutions are close to each other, but the closest solutions are obtained by the second-order schemes. 
The second-order finite difference schemes are constructed by original methods using shifted finite differences and integration of the filtration equations over grid cells.

The obtained numerical solutions can be used to prepare experiments and analyze the results of laboratory and field studies.

\section{References}

1. K.C. Khilar, H.S. Fogler, Migrations of fines in porous media (Dordrecht: Kluwer Academic Publishers, 1998).

2. C. Noubactep, S. Care, Dimensioning Metallic Iron Beds for Efficient Contaminant Removal, Chemical Engineering Journal, 163, 3 (2010) p. 454-460.

3. S. Bradford, H. Kim, B. Haznedaroglu, S. Torkzaban, S. Walker, Coupled factors influencing concentration-dependent colloid transport and retention in saturated porous media, Environmental Science and Technology, 43 (2009) p. 6996-7002.

4. D.C. Mays, J.R. Hunt, Hydrodynamic and chemical factors in clogging by montmorillonite in porous media, Environmental Science and Technology, 41 (2007) p. $5666-5671$.

5. C.V. Chrysikopoulos, V.I. Syngouna, Effect of gravity on colloid transport through water-saturated columns packed with glass beads: Modeling and experiments, Environmental Science and Technology, 48 (2014) p. 6805-6813.

6. A. Santos, P. Bedrikovetsky, Size exclusion during particle suspension transport in porous media: stochastic and averaged equations, Computational and Applied Mathematics 23, 2-3 (2004) p. 259-284.

7. J.P. Herzig, D.M. Leclerc, P. Legoff, Flow of suspensions through porous media application to deep filtration, Industrial and Engineering Chemistry, 62 (1970) p. 8-35.

8. P. Bedrikovetsky, Upscaling of stochastic micro model for suspension transport in porous media, Transport in Porous Media, 75 (2008) p. 335-369.

9. N.E. Leont'ev, D.A. Tatarenkova, Exact solutions to nonlinear equations of suspension flow through a porous medium, Moscow University Mechanics Bulletin, 70, 3 (2015) p. 61-65.

10. P. Bedrikovetsky, Z. You, A. Badalyan, Y. Osipov, L. Kuzmina, Analytical model for straining-dominant large-retention depth filtration, Chemical Engineering Journal, 330 (2017) p. 1148-1159.

11. D.N. Mikhailov, N.I. Ryzhikov, V.V. Shako, Experimental Investigation of Transport and Accumulation of Solid Particle and Clay Suspensions in Rock Samples, Fluid Dynamics, 50, 5 (2015) p. 691-704.

12. Y.P. Galaguz, G.L. Safina, Modeling of Particle Filtration in a Porous Medium with Changing Flow Direction, Procedia Engineering, 153 (2016) p. 157-161.

13. L.I. Kuzmina, Yu.V. Osipov, Y.P. Galaguz, A model of two-velocity particles transport in a porous medium, International Journal of Non-linear Mechanics, 93 (2017) p. 1-6.

14. E.F. Toro, Riemann solvers and numerical methods for fluid dynamics (Springer, Dordrecht, 2009)

15. Y.P. Galaguz, Realization of the TVD-scheme for a numerical solution of the filtration problem, International Journal for Computational Civil and Structural Engineering, 13, 2 (2017) p. 93-102. 